\title{
Arka and its pharmaceutical attributes in Indian alchemy (Rasashastra): A comprehensive review
}

\author{
Review Article
}

\author{
Anagha Vishwas Ranade ${ }^{1^{*}}$, Rabinarayan Acharya ${ }^{2}$
}

1. PhD scholar of Dravyaguna, 2. Professor, Dept. of Dravyaguna, IPGT\& RA, Gujarat Ayurved University, Jamnagar, Gujarat, India.

\begin{abstract}
Rasashastra, a branch of Ayurvedic system of medicine, deals with the pharmaceutical procedures of drugs of metallic, mineral and poisonous origin. These drugs are processed by some unique prescribed pharmaceutical techniques including Shodhana (purification process), Jarana (incineration) and Marana (calcination) that renders them fit for internal administration. Some specific groups of drugs of herbal origin viz. Marak gana (group of calcifying herbs), Dugdha varga (group of latex bearing plants), etc. are used for these procedures which primly include medicinal plants.

Arka, (Calotropis procera Ait. and Calotropis gigantea L.R.Br.) one of such plant from the semi-poisonous group finds manifold usage in these purification procedures and incineration techniques for many Rasaushadhis. Still, single hand information on the collective information regarding the use of Arka in various processing techniques in Rasashastra is deficient. Hence, a thorough review from available 37 texts related to Rasashastra was made and the necessary supplementary references of nano particles was found out through available resources from internet. After a critical analysis, a total of 133 references were found wherein the usage of Arka has been highlighted in different pharmaceutical processes in texts of Rasashastra. Among them, thirty eight are related to procedures of shodhana and marana of Parada (mercury), nineteen deal with procedures concerned with Maharasa, six and three respectively in case of Uparasa and Sadharana (mineral origin) rasa along with fifty five processing techniques of varied dhatus (metals).
\end{abstract}

Keywords: Ayurveda, Bhasma, Calotropis gigentia, Calotropis procera, Green chemistry, Nanotechnology

\section{Introduction:}

In Ayurveda, Rasashastra called as Alchemy is a prospective upcoming branch which deals with metals, minerals, animal drugs and poisonous drugs of plant origin. It has subtle roots in the past for effective treatment with typically nano-preparations i.e bhasmas. Main focus being Parada/Rasa (mercury),remaining drugs have been classified into Maha rasa, Uparasa, Sadharana rasa, Dhatu varga and Ratna varga.(1)In Rasashastra, all ingredients are first purified and reduced to their absolute essence in a process called Shodhana, where gems or metals are refined, through a process of alternate heating, cooling, oxidizing and crushing, to a fine pure ash. These individual purified ashes are called Bhasmas.(2) This processing is done with the help of triturating metals with different groups of drugs known as mishrak varga which probably assist in purification and gaining the absorbable particle size of metals particularly suitable in human body in form of medicines.

On a close analysis, plants categorised under semipoisonous group have been used in either shodhana (purification), bhavana (trituration) in marana (calcinations), etc to reduce metals to ash forms consisting

*Corresponding Author:

Anagha Vishwas Ranade

IPGT \& RA, Gujarat Ayurved University,

Jamnagar, Gujarat,

India. Email: anagharanade11@gmail.com of nano particles. Arka (Calotropis gigantea R.Br \& Calotropis procera Ait.) one among the semipoisonous group of plants, has been used commonly in these procedures. Classical texts of Ayurveda describe two types of Arka and Shwetarka/Alarka. Arka has three botanical sources. The possible correlation has been reported between classical types and botanical sources. (3) Its traditional and therapeutic and economic uses of Arka has been reported. It is also employed in preparing fireworks which also points to its use with chemicals that coincides with the traditional usage with metals and minerals.(4) The differential characters of white and purple variety of Calotropis gigantea has also been reported by micrometric evaluation of its flowers. (5) Calotropis procera and Calotropis gigantea have been studied extensively for its phytochemical (6),(7) and pharmacological profiles.(8),(9). Authors of various Rasagranthas have (texts possessing single and compound formulations containing mercury or metallic preparations) different opinion regarding the use of Arka in Shodhana/marana of Rasaushadhis. However, their references are scattered. Hence, it is the need of the time to present this comprehensive information on Arka so as to bring this valuable and frequently available drug into limelight. A thorough review from 37 texts related to Rasashastra was made and the necessary supplementary references of nano particles was found out through available 
resources from internet. The resultant information has been put forth in a tabulated manner consisting of the indication for which Arka has been incorporated and the exact process viz. Bhavana, kalka dravya, etc. alongwith its parts used. The Sanskrit terms of procedures have been expressed into convenient English language using Rasashastra texts. (10) Arka has also been incorporated in various mishrak vargas that are group of medicinal plants advocated for certain shodhana and marana procedures. The list has been compiled and presented in a systematic manner.

\section{Results:}

Arka was found to have place in twenty different vargas/ganas in thirty seven texts related to Rasashastra. Latex is included in most of them followed by leaf, flower and whole plant. (Table 1)

\begin{tabular}{|c|c|c|c|}
\hline Sr.no. & Part used & Varga/gana & References \\
\hline 1 & Kashtha (stem) & $\begin{array}{l}\text { Dantadhavana (group of plants useful } \\
\text { in dental cleansing) }\end{array}$ & $21,30,33,13$ \\
\hline 2 & Kshira (latex) & $\begin{array}{l}\text { Upavisha (semipoisonous group of } \\
\text { plants) }\end{array}$ & $\begin{array}{l}14,15,19,17,24,25,26,16,34,43,3 \\
5,36,37,46,44,41,40,30,32,43\end{array}$ \\
\hline 3 & Kshira (latex) & Dugdha varga (latex bearing plants ) & $15,19,34$ \\
\hline 4 & Kshira (latex) & Shringaberadi gana & 26,37 \\
\hline 5 & Kshira (latex) & $\begin{array}{l}\text { Dugdha vriksha gana (latex bearing } \\
\text { plants ) }\end{array}$ & 31,43 \\
\hline 6 & Kshira (latex) & $\begin{array}{l}\text { Abhraka marak gana (group of calci- } \\
\text { fying agents) }\end{array}$ & 34,43 \\
\hline 7 & Kshira (latex) & $\begin{array}{l}\text { Kshiratrayam (thrre plants bearing } \\
\text { plants) }\end{array}$ & 43,44 \\
\hline 8 & Kshira (latex) & $\begin{array}{l}\text { Abhraka putarha varga (group of in- } \\
\text { cinerating agents) }\end{array}$ & 36 \\
\hline 9 & Kshira (latex),patra (leaf) & $\begin{array}{l}\text { Parada marak varga group of calcify- } \\
\text { ing agents for mercury) }\end{array}$ & $26,34,37,46,44$ \\
\hline 10 & Mula (Root) & Vyaghrikadi gana & 15 \\
\hline 11 & Mula (root) & $\begin{array}{l}\text { Niyamak gana (group useful in pro- } \\
\text { cessing of mercury) }\end{array}$ & 43,37 \\
\hline 12 & Mula (root) & $\begin{array}{l}\text { Bhedaniya mahakashaya (drastic pur- } \\
\text { gative group) }\end{array}$ & 46,44 \\
\hline 13 & Mula (root) & $\begin{array}{l}\text { Svedopaga gana (group helping in } \\
\text { inducing diaphoresis) }\end{array}$ & 46,44 \\
\hline 14 & Mula (root) & Arkadi gana & 44,13 \\
\hline 15 & Mula (root) & $\begin{array}{l}\text { Vamanopaga (group helping in in- } \\
\text { ducing emesis ) }\end{array}$ & 44 \\
\hline 16 & Mula (root) & $\begin{array}{l}\text { Shrovirechanakari gana ( group use- } \\
\text { ful in nasya karma) }\end{array}$ & 13 \\
\hline 17 & Mula (root) & $\begin{array}{l}\text { Vatanashan gana (group of allevia- } \\
\text { tion of Vata dosha) }\end{array}$ & 13 \\
\hline 18 & Mula (root) & Adhobhagahar gana & 13 \\
\hline 19 & Panchanga (whole plant) & $\begin{array}{l}\text { Ksharashtaka (group containing } \\
\text { plants producing alkalis) }\end{array}$ & $14,43,32$ \\
\hline 20 & Panchanga (whole plant) & $\begin{array}{l}\text { Kshara varga (group containing } \\
\text { plants producing alkalis) }\end{array}$ & 15 \\
\hline 21 & Patra(leaves) & Shringaberadi gana & 44 \\
\hline 22 & Pushpa (Flower) & $\begin{array}{l}\text { Kaphanashak gana (group alleviating } \\
\text { kapha) }\end{array}$ & 43 \\
\hline 23 & Pushpa (Flower) & $\begin{array}{l}\text { Pushpa varga (group of therapeutic } \\
\text { use of flowers) }\end{array}$ & 42 \\
\hline
\end{tabular}


Use of Arka in various Rasashastra texts for Shodhana (purification procedure), Marana (caicination) and Jarana (incineration) of different Rasa drugs are presented in table 2 . After a critical analysis, a total of 133 references have been found wherein the usage of Arka has been highlighted in 37 different texts of Rasashastra. Among them, thirty eight are related to procedures of shodhana and marana of Parada (mercury), nineteen deal with procedures concerned with Maharasa, six and three respectively in case of Uparasa and Sadharanarasa. Arka finds place extensively in fifty five processing techniques of varied dhatus (metals) whereas it is present in eight methods of processing of ratnas (gems). Meagre indications of Arka are also denoted in case of jangam dravya (drugs of animal origin) mrigashringa bhasma (calcinated ash of a special type of deer's horn) and in formation of calcinated preparation of silica (kacha). The use of Shweta variety of Arka has been specified in 6 methods of Parada (mercury) purification and incineration. Parts used include kshira(latex) in particular followed by leaf juice, decoction etc.

\begin{tabular}{|c|c|c|c|c|}
\hline \multicolumn{5}{|c|}{$\begin{array}{c}\text { Table 2: Use of Arka in various Rasashastra texts for Shodhana (purification procedure), Marana } \\
\text { (caicination) and Jarana (incineration) of different Rasa drugs }\end{array}$} \\
\hline Sr. no & Part used & Indication & Bhavana /kalka/anupana & References \\
\hline & \multicolumn{4}{|l|}{ Parada prakaran } \\
\hline 1 & Mula (root) & Bijottara kriya & Bhavana (trituration) & 40 \\
\hline 2 & Mula (root) & Bubhuksha vidhi & Bhavana & 36,46 \\
\hline 3 & Kshira (latex) & $\begin{array}{l}\text { Garbha druti (internal } \\
\text { liquefaction) }\end{array}$ & Bhavana & 12,14 \\
\hline 4 & Kshira (latex) & Grasa vidhi & Bhavana & 37 \\
\hline 5 & Kshira (latex) & Khota jarana & Bhavana & 14 \\
\hline 6 & Kshira (latex) & Khota nirmana & Bhavana & 14 \\
\hline 7 & Kshira (latex) & Madana mudra & Bhavana & 34 \\
\hline 8 & Panchanga(whole plant) & Mridu dravya arka vidhi & Kalka (paste) & 31,33 \\
\hline$\overline{9}$ & Mula (root) & Mulika bandha & Bhavana & 18,21 \\
\hline 10 & Phala (fruit) & Mulika bandha & Bhavana & 21 \\
\hline 11 & Kshira (latex) & Nigal parada with lavana & Bhavana & 14 \\
\hline 12 & Kshira (latex) & Nigal parada with loha & Bhavana & 14 \\
\hline 13 & Kshira (latex) & Nigalbaddha parada & Bhavana & $14,25,21,33$ \\
\hline 14 & Kshira (latex) & Nigalottama parada & Bhavana & 14 \\
\hline 15 & Patra(leaves) & Parad pujan & - & 21 \\
\hline 16 & Mula (root) of white variety & $\begin{array}{l}\text { Parada anuvasana } \\
\text { (rehabilitation) }\end{array}$ & Bhavana & 37 \\
\hline 17 & Kshira (latex) & $\begin{array}{l}\text { Parada bandhana } \\
\text { (bonding) }\end{array}$ & Bhavana & 37,47 \\
\hline 18 & Kshira (latex) & Parada bhasma & Bhavana & 37,43 \\
\hline 19 & Kshira (latex) & Parada garbha druti & Bhavana & 21 \\
\hline 20 & Kshira (latex) & $\begin{array}{l}\text { Parada jarana } \\
\text { (exhaustion) }\end{array}$ & Bhavana & $19,37,39$ \\
\hline 21 & Patra(leaves) juice & $\begin{array}{l}\text { Parada jarana- abhrajirna } \\
\text { parada sevana }\end{array}$ & Anupana (vehicle) & 15 \\
\hline 22 & Kshira (latex)(white) & Parada marana & Bhavana & 14 \\
\hline 23 & Kshira (latex) & $\begin{array}{l}\text { Parada marana- rasa bija } \\
\text { nirmana }\end{array}$ & Bhavana & 12 \\
\hline 24 & Kshira (latex) & $\begin{array}{l}\text { Parada murcchana } \\
\text { (swooning) }\end{array}$ & Bhavana & $24,46,21,33$ \\
\hline 25 & Kshira (latex) & Parada sancharana & Bhavana & 14 \\
\hline 26 & $\begin{array}{l}\text { Patra(leaves) juice of white } \\
\text { variety }\end{array}$ & $\begin{array}{l}\text { Parada sandipana } \\
\text { (stimulation) }\end{array}$ & Bhavana & 47 \\
\hline 27 & Kshira (latex) & Parada sanskar & Bhavana & 20 \\
\hline 28 & Kshira (latex) & Parada shodhana & Bhavana & 29 \\
\hline 29 & $\begin{array}{l}\text { Patra(leaves) juice of white } \\
\text { variety }\end{array}$ & $\begin{array}{l}\text { Parada svedana } \\
\text { (steaming) }\end{array}$ & - & 19 \\
\hline 30 & Mula (root) & Parada svedana & Bhavana & 39 \\
\hline
\end{tabular}


Anagha Vishwas Ranade et.al., Review on use of Arka in procedures of Rasashastra

\begin{tabular}{|c|c|c|c|c|}
\hline 31 & Mula (root) powder & $\begin{array}{l}\text { Parada urdhvapatana } \\
\text { (upward sublimation) }\end{array}$ & Dravya & 33 \\
\hline 32 & Kshira (latex) & Parada yukta bhasma & Bhavana & 16 \\
\hline 33 & Kshira (latex) & Uttama bandhana & Bhavana & 21 \\
\hline 34 & Kshira (latex) & Utthapana sanskar & Bhavana & 22 \\
\hline 35 & Kshira (latex) & Vadavanala bida & Bhavana & $25,16,21$ \\
\hline 36 & Kshira (latex) & Vaishvanar bida & Bhavana & 21 \\
\hline 37 & Kshira (latex) & Vajranala bida & Bhavana & 21 \\
\hline 38 & $\begin{array}{l}\text { Patra(leaves) decoction of } \\
\text { white variety }\end{array}$ & Varnakarana & anupana & 14 \\
\hline \multicolumn{5}{|c|}{ Maharasa } \\
\hline 39 & Mula(root) juice & Abhrak bhasma & Bhavana & $25,26,21$ \\
\hline 40 & Kshira (latex) & Abhraka bhasma & Bhavana & $\begin{array}{l}23,25,27,16,35,4 \\
6,44,33\end{array}$ \\
\hline 41 & Kshira (latex) & Abhraka charana vidhi & Bhavana & 21 \\
\hline 42 & Kshira (latex) & Abhraka druti(liquefaction) & Bhavana & 34,37 \\
\hline$\overline{43}$ & Kshira (latex) & Abhraka druti & Bhavana & 37 \\
\hline 44 & $\begin{array}{l}\text { Patra(leaves), mula(root), } \\
\text { kshira (latex) }\end{array}$ & Abhraka marana & Bhavana, veshtana & $\begin{array}{l}25,26,16,43,37,2 \\
1,30,46,43\end{array}$ \\
\hline 45 & Kshira (latex) & Abhraka marana & Bhavana & $\begin{array}{l}34,43,36,46,38,4 \\
7,45\end{array}$ \\
\hline 46 & Kshira (latex) & Abhraka nishchandratva & Bhavana & 37 \\
\hline 47 & Kshira (latex) & Abhraka satvapatana & Bhavana & 14 \\
\hline 48 & Patra(leaves) & Abhraka satvapatana & Bhavana & 21 \\
\hline 49 & Patra(leaves) & Abhraka shodhana & Bhavana & 25 \\
\hline 50 & Kshira (latex) & Abhraka shodhana & Bhavana & 21 \\
\hline 51 & Kshira (latex) & Dhanyabhraka marana & Bhavana & $\begin{array}{l}20,17,26,29,34,3 \\
0\end{array}$ \\
\hline 52 & Kshira (latex) & Hemavajra lepana & Lepana & 21 \\
\hline 53 & Kshira (latex) & Makshik marana & Bhavana & 37 \\
\hline 54 & Kshira (latex) & Makshika satvapatana & Bhavana & $11,14,21$ \\
\hline 55 & Kshira (latex) & Rasaka satvapatana & Bhavana & 40 \\
\hline 56 & Kshira (latex) & Svarna makshika shodhana & Bhavana & 34 \\
\hline 57 & Kshira (latex) & Vaikranta satvapatana & Bhavana & $16,46,33$ \\
\hline \multicolumn{5}{|c|}{ Uparasa } \\
\hline 58 & Kshira (latex) & Hartala satvapatana & Bhavana & $15,34,43,37,21$ \\
\hline 59 & Kshira (latex) & Hartala marana & Bhavana & $16,43,37,46,45$ \\
\hline 60 & Patra(leaves) juice & Hartala marana & Bhavana & 35 \\
\hline 61 & Kshira (latex) & Hartala bhasma & Bhavana & 36,33 \\
\hline 62 & Kshira (latex) & Manashila marana & Bhavana & 44 \\
\hline 63 & Kshira (latex) & $\begin{array}{l}\text { Sarva uparasa } \\
\text { satvapatana }\end{array}$ & Bhavana & 19 \\
\hline \multicolumn{5}{|c|}{ Sadharana rasa } \\
\hline 64 & Phala (fruit) & Hingula marana & Bhavana & 16 \\
\hline 65 & Kshira (latex) & Hingula shodhana & Bhavana & 35 \\
\hline 66 & Kshira (latex) & Shankhiya maran & Bhavana & 35 \\
\hline \multicolumn{5}{|c|}{ Dhatu } \\
\hline 67 & Kshira (latex) & Dhatu marana & Bhavana & $25,43,37,41,30$ \\
\hline 68 & Kshira (latex) & Dhatu shodhana & Bhavana & 27 \\
\hline 69 & Kshira (latex) & $\begin{array}{l}\text { Ghoshakrushta tamra } \\
\text { vedhana }\end{array}$ & Bhavana & 12 \\
\hline
\end{tabular}


International Journal of Ayurvedic Medicine, 2015, 6(4), 280-288

\begin{tabular}{|c|c|c|c|c|}
\hline 70 & Kshira (latex) & Guhya naga & Bhavana & $14,15,21$ \\
\hline 71 & Kshira (latex) & Hema karana & Bhavana & 18 \\
\hline 72 & Kshira (latex) & Hemavati vidya & Bhavana & 16 \\
\hline 73 & $\overline{\text { Kshira (latex) }}$ & Kansya bhasma & Bhavana & 46 \\
\hline 74 & Kshira (latex) & Kansya marana & Bhavana & 44 \\
\hline 75 & Kshira (latex) & Kansya pittala bhasma & Bhavana & 16,33 \\
\hline 76 & Kshira (latex) & Kansya pittala shodhana & Bhavana & 34 \\
\hline 77 & Kshira (latex) & Kanta loha shodhana & Bhavana & 16,33 \\
\hline 78 & Kshira (latex) & Loha bhasma & Bhavana & 36 \\
\hline 79 & Kshira (latex) & Loha jarana & Bhavana & 25 \\
\hline 80 & Kshira (latex) & Loha marana & Bhavana & $\begin{array}{l}11,17,16,34,36, \\
37,46\end{array}$ \\
\hline 81 & Kshira (latex) & loha shodhana & Bhavana & $43,35,30$ \\
\hline 82 & Kshira (latex) & loha shodhana & Nirvapana & 33 \\
\hline 83 & Kshira (latex) & Mandura marana & Bhavana & 45 \\
\hline 84 & Kshira (latex) & Naga bhasma & Bhavana & 19,36 \\
\hline 85 & Mula (root) & Naga bhasma & Stirring & 36 \\
\hline 86 & Kshira (latex) & $\begin{array}{l}\text { Naga bhasma with hema } \\
\text { bija }\end{array}$ & Bhavana & 21 \\
\hline 87 & Kshira (latex) & Naga marana & Bhavana & 43,37 \\
\hline 88 & Patra (leaves) juice & Naga marana & Bhavana & 37 \\
\hline 89 & Kshira (latex) & Naga shodhana & Nirvapana & $\begin{array}{l}17,23,25,26,16, \\
35,37,46,32\end{array}$ \\
\hline 90 & Kshira (latex) & Nagarahit bhasma & Bhavana & 34 \\
\hline 91 & Kshira (latex) & Nagasambhuta Chapala & Bhavana & $14,15,34,44$ \\
\hline 92 & Kshira (latex) & Panch loha marana & Bhavana & $16,46,33$ \\
\hline 93 & Kshira (latex) & Panch loha shodhana & Bhavana & 34 \\
\hline 94 & Kshira (latex) & Pittala marana & Kalka dravya & 17,37 \\
\hline 95 & Kshira (latex) & Rajata bhasma & Bhavana & 23 \\
\hline 96 & Kshira (latex) & Rajata marana & Bhavana & $26,46,32$ \\
\hline 97 & Kshira (latex) & Raupya karana & Bhavana & $18,22,37$ \\
\hline 98 & Kshira (latex) & Sarva loha shodhana & Nirvapana & $14,34,21$ \\
\hline 99 & Kshira (latex) & $\begin{array}{l}\text { Sarva pashana } \\
\text { satvapatana }\end{array}$ & Bhavana & 27 \\
\hline 100 & Patra (leaves) juice & Sarva pashana shodhana & Bhavana & 27 \\
\hline 101 & Patra (leaves) juice & Svarna bhasma & Bhavana & 23,16 \\
\hline 102 & Kshira (latex) & Svarna bhasma & Bhavana & 36,37 \\
\hline 103 & Kshira (latex) & Svarna jarana & Bhavana & 14 \\
\hline 104 & Kshira (latex) & Svarna marana & Bhavana & $\begin{array}{l}17,26,16,34,43, \\
46,33\end{array}$ \\
\hline 105 & Patra (leaves) juice & Svarna marana & Bhavana & 46 \\
\hline 106 & Kshira (latex) & Svarna shodhana & Bhavana & 21,33 \\
\hline 107 & Kshira (latex) & Svarna shodhana & Nirvapana & 33 \\
\hline 108 & Patra (leaves) & Tamra bhasma & Acchadana & 20 \\
\hline 109 & Kshira (latex) & Tamra bhasma & Bhavana & $20,16,36,46,33$ \\
\hline 110 & Kshira (latex) & $\begin{array}{l}\text { Tamra bhasma } \\
\text { nirutthikarana }\end{array}$ & Bhavana & 36,46 \\
\hline 111 & Kshira (latex) & Tamra marana & Bhavana & 29,44 \\
\hline 112 & Kshira (latex) & Tamra shodhana & Bhavana & $\begin{array}{l}14,19,25,26,16, \\
43,37,44,38,21, \\
30,33\end{array}$ \\
\hline
\end{tabular}


Anagha Vishwas Ranade et.al., Review on use of Arka in procedures of Rasashastra

\begin{tabular}{|c|c|c|c|c|}
\hline 113 & Patra (leaves) juice & Tamra shodhana & Bhavana & 33 \\
\hline 114 & $\begin{array}{l}\text { Kshira (latex) of white } \\
\text { variety }\end{array}$ & Tamra shodhana & Bhavana & 14 \\
\hline 115 & Kshira (latex) & $\begin{array}{l}\text { Tamra shodhana- bija } \\
\text { vidhan }\end{array}$ & Bhavana & 12 \\
\hline 116 & Kshira (latex) & Tara nirmalikarana & Bhavana & 14 \\
\hline 117 & Patra (leaves) juice & $\begin{array}{l}\text { Tutthottha tamra } \\
\text { shodhana }\end{array}$ & Bhavana & 36 \\
\hline 118 & Kshira (latex) & $\begin{array}{l}\text { Vanga bhasmawith hema } \\
\text { bija }\end{array}$ & Bhavana & 21 \\
\hline 119 & Kshira (latex) & Vanga marana/bhasma & Bhavana & $\begin{array}{l}15,19,26,26,16, \\
43,36,37,46,44, \\
40,21,33\end{array}$ \\
\hline 120 & Kshira (latex) & Vanga shodhana & Bhavana & 11,35 \\
\hline 121 & Kshira (latex) & Vanga shodhana & Nirvapana & $\begin{array}{l}17,23,25,26,16, \\
43,37,44,32\end{array}$ \\
\hline 122 & Kshira (latex) & Yashada shodhana & Bhavana & 35 \\
\hline \multicolumn{5}{|c|}{ Ratna } \\
\hline 123 & Kshira (latex) & $\begin{array}{l}\text { Kshatriya vajra bhasma } \\
\text { vidhi }\end{array}$ & Bhavana & 14,37 \\
\hline 124 & Kshira (latex) & Ratna dravan vidhi & Bhavana & 14 \\
\hline 125 & Kshira (latex) & Ratna druti & Bhavana & $15,18,19,37$ \\
\hline 126 & Kshira (latex) & Samanya shodhana & Bhavana & 34 \\
\hline 127 & Kshira (latex) & Vaishya vajra marana & Bhavana & 34,37 \\
\hline 128 & Kshira (latex) & Vajra dravikarana & Bhavana & 14 \\
\hline 129 & Kshira (latex) & Vajra druti & Bhavana & 21 \\
\hline 130 & Kshira (latex) & Vajra marana, ranjana & Bhavana & 11,21 \\
\hline \multicolumn{5}{|c|}{ Miscellaneous } \\
\hline 131 & Kshira (latex) & Kacha bhasma & Bhavana & 36 \\
\hline \multicolumn{5}{|c|}{ Jangam dravya } \\
\hline 132 & Kshira (latex) & Mrigashringa marana & Bhavana & 43 \\
\hline 133 & Kshira (latex) & Mrigashringa bhasma & Bhavana & 41 \\
\hline
\end{tabular}

\section{Discussion:}

It is observed that different parts of both the varieties of Arka have been advocated in Shodhana, Marana, Bhasmikarana, Satvapatana, Bida nirmana, Druti and Bandhana of Parada and other metals and minerals.(Table 3)

\begin{tabular}{|l|l|l|}
\hline \multicolumn{3}{|c|}{ Table 3: Use of Arka in various processes of Rasa aushadhis } \\
\hline Procedure & Drug name(metal/mineral) & Part used \\
\hline $\begin{array}{l}\text { Shodhana (purifactory } \\
\text { procedures) }\end{array}$ & $\begin{array}{l}\text { Parada,Abhraka,Hingula,Vanga, Naga, Loha, Svarna, } \\
\text { Tamra, Yashada, Kansya, Pittala, Panchaloha, Ratna }\end{array}$ & Kshira(latex), Patra (leaves). \\
\hline $\begin{array}{l}\text { Marana } \\
\text { calcinations) }\end{array}$ & $\begin{array}{l}\text { Parada, Svarna Makshika, Abhraka, Hartala, } \\
\text { Manashila, Hingula, Shankhiya, Vanga, Naga, Loha, } \\
\text { Svarna, Rajata, Tamra, Mandura, Pittala, Kansya, } \\
\text { Vajra, Mrigashringa }\end{array}$ & $\begin{array}{l}\text { Mula(root) } \\
\text { Bhasmikarana } \\
\text { Rajada, Patra (leaves), }\end{array}$ \\
\hline Satvapatana, Hartala, Naga, Vanga, Loha, Svarna, & Kshira(latex), Patra (leaves) \\
\hline Bida nirmana & Abhraka, Makshik, Vaikranta, Hartala & Kshira(latex) \\
\hline Druti (liquefaction) & Vadavanala, Vaishvanar, Vajranala & Kshira(latex) \\
\hline $\begin{array}{l}\text { Bandhana (mercurial } \\
\text { bonding) }\end{array}$ & Parada, Mulika, Uttama, Khota & Kshira (latex) \\
\hline
\end{tabular}


Among the parts used, kshira (latex) has been utilized maximum in all the methods of shodhana and marana followed by leaf, root, fruit, flower and whole plant. Latex has also been the leading parts used for trituration process i.e bhavana and nirvapana (dipping). This widespread use of Calotropis latex highlights its significance in formation of bhasmas in particular.

Bhavana (trituration) is a unique pharmaceutical process in which a drug or mixture of drugs in powdered form is triturated with sufficient quantity of liquid media [viz. plant extractives (expressed juice, decoction etc) or animal products (urine, milk etc)] till liquid portion gets absorbed completely. Bhavana facilitates in mixing of ingredients of mixture for Bhavana and may account several chemical interactions in between them. Probable frequent alterations in particle size during Bhavana and reduction at the end may increase absorption and improve bioavailability of the drug.(48)

Bhasmas are often equated with nano particle technology. But there were certain debatable issues regarding the specific sequential processing in the different plant material like juices, latex, decoctions, etc. for bhasma production that increase the bioavailability of the formulation which was quoted to be distinct than the conventional synthetic methods of developing nanoparticles from metals. But recently, a concept of green synthesis of nano particles of different metals has emerged that has bridged a gap between conventional and Ayurvedic pharmaceutics. This includes biosynthetic green method that involves use of medicinal plants in manufacture of nano particles. Many references are available wherein Calotropis latex has been used in such technique. Silver nanoparticles were successfully derived by subjecting it to green synthesis that used serum of Calotropis latex. (49) This confirms the traditional claim of Arka latex in Rajata (silver) shodhana, marana. Harne et al. synthesized copper nanoparticle using the aqueous extract of latex of Calotropis procera L. and showed its excellent long term stability. A microwave-mediated simple and rapid method of gold nanoparticles (GNPs) synthesis using latex of Calotropis procera has been reported (50). Fourier transform infrared (FTIR) analysis indicated the presence of organic coating on the nanoparticles. Cytotoxicity of the GNPs was tested on HeLa and A549 and found to be nontoxic which was indicating that latex of Calotropis procera provided the nontoxic coating on GNPs, thus can be used as biomedical and pharmacological applications. Similarly, the rapid biological synthesis of zinc nanoparticles using leaf extract of Calotropis gigantea provides an environmental friendly, simple and efficient route for synthesis of nanoparticles. (51) Calotropis procera $L$. latex were used to fabricate $\mathrm{Cu} \mathrm{Nps}$ from copper acetate and transmission electron microscopy (TEM) revealed the average size was found to be $15 \pm 1.7 \mathrm{~nm}$.(52) Cysteine proteases (proteolytic enzymes) present in the latex act as capping agent and contributed to long term stability of $\mathrm{Cu}$ Nps (6 months) in aqueous medium.(53) The use of plant extracts avoids the usage of harmful and toxic reducing and stabilizing agents. Thus, this re- confirms the traditional claim of use of Arka kshira, patra, etc. in Svarna (gold) bhasma, Rajata (silver) bhasma, Tamra(copper) bhasma and Yashada (zinc carbonate) bhasma.

\section{Conclusion}

Arka is one of the frequently used herbal drug in Rasashastra for various procedures. There are more than 133 references where thirty eight are related to procedures of shodhana and marana of Parada (mercury), nineteen deal with procedures concerned with Maharasa, six and three respectively in case of Uparasa and Sadharanarasa along with fifty five processing techniques of varied dhatus (metals). Arka is also used extensively i.e. more than in eight methods of processing of ratnas (gems). All most all the parts used of Arka are used for various procedures of rasashastra. However, latex is used more frequently and coincides with the modern green synthesis of particles.

\section{References}

1. Angadi Ravindra. A textbook of Rasasastra. Varanasi; Chaukhambha Surabharati Prakashan; 2014. Pg3.

2. Gomes A, Ghosh S, Sengupta J, Datta P, Gomes A. Herbonanoceuticals: A New Step Towards Herbal Therapeutics. Med Aromat Plants 2014; vol 3(3); 162.

3. Ranade Anagha V, AcharyaRabinarayan. Arka and its botanical equivalents: A critical analysis. Ayurpharm Int J Ayur Alli Sci. 2015;4(3); p 60-68.

4. Ranade Anagha, Acharya Rabinarayan. An Appraisal On Ethno-Medicinal Claims Of Calotropis Procera Ait And Calotropis Gigantea (Linn) R.Br. Two Source Drugs Of Ayurvedic Medicinal Plant 'Arka'. Global J Res. Med. Plants \& Indigen. Med. (2014); Volume 3(12); p 475-488.

5. Ranade Anagha, Harisha CR, Acharya Rabinarayan and Jani Switu. Micro-Morphological and Micrometric evaluation of White and Purple variety Flowers of Calotropis gigantea Linn. R.Br. IJUPBS. Sept-Oct 2014; 3(5); 289-302.

6. V. Saratha, S. Iyyam Pillai and S. Subramania. Isolation and characterization of lupeol in Calotropis gigantea latex. International Journal of Pharmaceutical Sciences review and Research. 2011; Volume10 (2); 54-57.

7. Sheth Falguni. Range of seasonal and phytochemical variations in Calotropis procera Ait.Int.J.Med.Aromatic Plants, 2011;Volume 1 (2);180-183.

8. Bulani Vipin et al. Inhibitory effect of Calotropis gigantea extract Ovalbumin-induced airway inflammation and Arachidonic acid induced inflammation in a murine model of asthma. International Journal of Current Biological and Medical Science. 2011; 1(2); 19- 25.

9. Kumar Suresh P, Suresh E \& S. Kalavathy. Review on a potential herb Calotropis gigantea (L.) R. Br. Scholars Academy of Journal of Pharmacy. 2013; 2 (2); 135-143. 
10.Murthy Himasagara Chandra. Rasasastra -The Mercurial system. $1^{\text {st }}$ edition. Varanasi; Chaukhambha Sanskrit series. 2008

11.Nagarjuna. Rasendramangal. Sharma H S. editor.1st edi.Varanasi; Chaukhambha orientalia Prakashan. 2003.

12.Bhagvatpada Govinda. Rasa Hriday Tantra. Shastri daulatram. $3^{\text {rd }}$ edi. Varanasi; Chaukhambha orientalia prakashan. 2005.

13.Acharya Vangasen. Vangasen. First edition. Varanasi; Chaukhambha Sanskrit series. 2009.

14.Tripathi Indradeo editor. Rasarnaav naam rasatantram. $4^{\text {th }}$ edi.Varanasi; Chaukhambha Sanskrit series. 2001.

15.Somadev. Rasendrachudamani. Mishra Siddhinandan editor. First edition. Varanasi; Chaukhambha orientalia prakashan. Reprint 2009.

16.Madhav. Ayurveda prakash. Mishra Gulrajsharma.editor. First edition. Varanasi; Chaukhambha Bharati academy. Reprint 2007.

17.Sharangdhar samhita with Gudharthadipika and dipika commentary. Parshuramshastri Vidyasagar. First edition. Varanasi; Krishnadas academy. Reprint 2000.

18.Yashodhara. Rasa prakash sudhakar. Mishra Siddhinandan editor. First edition. Varanasi; Chaukhambha orientalia prakashan. Reprint 2009.

19. Vagbhata.

Rasaratnasamucchaya.

Shastri Ambikadutta. $9^{\text {th }}$ edi. Varanasi; Chaukhambha Amarabharati Prakashan. 2010.

20.Bindu. Rasa paddhati. Mishra Siddhinandan editor. Varanasi; Chaukhambha orientalia prakashan. 2005.

21.Bhairav. Anandakanda. Mishra Siddhinandan.editor. $2^{\text {nd }}$ edi. Varanasi; Chaukhambha orientalia prakashan. first edi.2008.

22.Anant dev suri. Rasachintamani. Mishra Siddhinandan editor. First edition.Varanasi; Chaukhambha orientalia prakashan.1990.

23. Shalinath. Rasamanjiri. Mishra Siddhinandan editor. First edition. Varanasi; Chaukhambha orientalia prakashan. 1995.

24.Kayastha Chamunda. Rasa Sanket Kalika. Shashtri Satyanarayan pammi. First edition Varanasi; Chaukhambha krishnadas academy.2005.

25.Dhundhukanath. Rasendrachitamani. Mishra Siddhinandan editor. First edition. Varanasi; Chaukhambha orientalia prakashan. 2000.

26.Bhatt Gopalkrishna. Rasendrasara samgraha. Ramtej Pandya edi. First edition. New delhi; Chaukhambha Sanskrit pratitshthan. Reprint 2010.

27. Sharma Dnyanachandra. Rasa kaumudi. Sharma Pavani Prasad. Editor. First edition .Varanasi; Chaukhambha Vidya Bhavan. 1966.

28.Mishra Chudamani. Rasa Kamadhenu. Mishra Gulrajsharma. Editor. $2^{\text {nd }} \quad$ edi.Varanasi; Chaukhambha orientalia. 1999.

29.Trimalla bhat. Yogatarangini. Charantirtha Maharaja. Editor. First edition. Gondal; Saurashtra. Rasashala Aushadhashrama.1956.
30.Shastri lakshmipati. Yogaratnakar. First edition.Varanasi; chaukhambha Prakashana. Reprint 2010.

31.Lankapati ravan. Arka prakash. Tripathi Indradeo. Editor. First edition.Varanasi; Krishnadas academy. 1995.

32.Govindadasa. Bhaishyajya ratnavali. Shastri Ambikadutta. Editor. First edition. Varanasi; Chaukhambha prakashan. Reprint 2011.

33.Panshikar VL, Soman KV. Nighanta ratnakar. First edition. Varanasi; Chaukhambha sansthan Prakashan. 2011.

34.Chaubhe Dattaram. Brihat Rasaraja sunadara. $3^{\text {rd }}$ edition. Varanasi; Chaukhambha orientalia prakashan. $3^{\text {rd }}$ edition. 2000.

35.Bhagat Bhagwandas. Rasaraja mahodadhi. First edition. Mumbai; Khemraj Shrikrishnadas Prakashan. 2010.

36. Vaishya shamsundaracharya. Rasayanasara. First edition. Varanasi; Chaukhambha Krishnadas academy. 2005. Vol I \& II.

37. Mukherjee Bhoodeb. Rasa jala nidhi. $4^{\text {th }}$ edition. New Delhi; Chaukhambha Orientalia prakashan. 2004. Vol I - VolV.

38. Acharya Jadavji trikamji. Rasmritam. Joshi damodar. Editor. First edition. Varanasi; Chaukhmabha Sanskrit bhavan. 1998.

39.Bhatt Krishnaram. Siddhabheshaja manimala. $3^{\text {rd }}$ edition. Varanasi; Chaukhambha Krishnadas academy.2003.

40.Sharma Badrinath. Rasopanishat. First edition. Kaleda, Ajmer; Krishna Gopal Mudranalaya. 1959.

41.Sharma Trimbaknath. Rasamitra. First edition. Varanasi; Chaukhambha Sanskrit series. Reprint 2001.

42.Panditrao DD. Sahasrayogam. First edition. New Delhi; CCRAS. Yugantar prakashan. 1990.

43.Mishra Sadanand. Rasatarangini. Shastri Kashinath.editor. $11^{\text {th }}$ edition.Delhi; Motilala banarasidas. 2009.

44.Dwivedi Vishwanath. Rasendra sambhav. First edition.Varanasi; Krishnadas academy. 1997.

45.Acharya Vishram. Anupana manjiri. First edition. Jamnagar. Sahitya sanshodhan Vibhagiya Prakashan. Gujarat Ayurved University. 1972.

46.Shah Nagindas Chhanganlal. Bharat Bhaishyajya Ratnakar. First edition. New Delhi; B.Jain Publishers. 2005. Vol I-Vol V.

47.Bajpai Rameshwar Dayal. Rasa dipika. First edition. Varanasi; Chaukhmbha Krishnadas academy.2003.

48.Sharma Rohit, Prajapati PK. Liquid media's in Bhavana Samskara: A pharmaceutico-therapeutic prospect.The Journal of Phytopharmacology. 2015; 4 (1); 49-57.

49.Nadia Hussein Mohamed, Mady Ahmed Ismail, Wael Moustfa Abdel-Mageed, Ahmed Abdelfattah Mohamed Shoreit. Antimicrobial activity of latex silver nanoparticles using Calotropis procera. Asian Pac J trop Biomed.2014; 4(11); 876-883.

50.Ratul Kumar Das, Punuri Jayasekhar Babu, Nayanmoni Gogoi, Pragya Sharma, and Utpal Bora. Microwave-Mediated Rapid Synthesis of Gold 
Nanoparticles Using Calotropis procera Latex and Study of Optical Properties. Nanomaterials. Volume 2012 (2012).

51.Vidya $\mathrm{C}$ et al. Green synthesis of $\mathrm{ZnO}$ nanoparticles by Calotropis Gigantea. International Journal of Current Engineering and Technology, 2013; Special Issue 1; 118-120.

52.Shobha G, Vinutha Moses \& Ananda S.Biological Synthesis of Copper Nanoparticles and its impact - a
Review.International Journal of Pharmaceutical Science Invention.2014; 3(8);28-38.

53.S. Harne, A. Sharma, M. Dhaygude, S. Joglekar, K. Kodam and M. Hudlikar. Novel route for rapid biosynthesis of copper nanoparticles using aqueous extract of Calotropis procera L. latex and their cytotoxicity on tumor cells. Colloids Surf B Biointerfaces. 2012,;15 (95);284-288. 